\title{
CHARACTERIZATION OF CERTAIN RIEMANN SPACES BY DEVELOPMENT
}

\section{MINORU KURITA}

The purpose of this paper is to characterize the Riemann space whose line element is given by

$$
d s^{2}=a\left(x^{i}\right)^{2} g_{\alpha \beta}\left(x^{\Upsilon}\right) d x^{\alpha} d x^{\beta}+g_{\lambda \mu}\left(x^{i}\right) d x^{\lambda} d x^{\mu} .
$$

Through the whole description we use the indices $i, j, \alpha, \beta, \gamma, \lambda, \mu, \nu$ when they run as follows

$$
\begin{aligned}
& i, j \quad=1,2, \ldots, n \\
& \alpha, \beta, \gamma=1,2, \ldots, k \\
& \lambda, \mu, \nu=k+1, k+2, \ldots, n
\end{aligned}
$$

and $a\left(x^{i}\right)$ and $g_{\lambda \mu}\left(x^{i}\right)$ shall mean to be functions of $x^{1}, \ldots, x^{n}, g_{\alpha \beta}\left(x^{\top}\right)$ a function of $x^{1}, \ldots, x^{k}$ and $g_{\lambda \mu}\left(x^{\nu}\right)$ a function of $x^{k+1}, \ldots, x^{n}$. This Riemann space (1) contains as special cases many important spaces such as a directly decomposable space, a conformally separable space and a space with torse-forming vector field. K. Yano [1] characterized a conformally separable Riemann space by umbilical surfaces contained in it and the proof of Theorem 1 of [1] leads to the following:

THEOREM. The necessary and sufficient condition for an n-dimensional Riemann space to have an arc-element given by (1) is that it has $n$-k-parametric family of $k$-dimensional totally umbilic surfaces and $k$-parametric family of $n-\dot{k}$ dimensional surfaces which are orthogonal to the former.

We characterize this space (1) from another point of view.

1. We begin by determining the Riemannian connection, namely the euclidean connection without torsion, attached to (1). We can take $k$ Pfaffians

$$
\omega_{\alpha}=p_{\alpha \beta}\left(x^{\curlyvee}\right) d x^{\beta}
$$

such that

$$
g_{\alpha \beta}\left(x^{\gamma}\right) d x^{\alpha} d x^{\beta}=\sum\left(\omega_{\alpha}\right)^{2}
$$

Received April 24, 1951. 
Then, according to E. Cartan, Pfaffians $\omega_{\alpha \beta}$ satisfying the relations

$$
\omega_{\alpha}^{\prime}=\left[\omega_{\beta} \omega_{\beta \alpha}\right], \quad \omega_{\alpha \beta}=-\omega_{\beta \alpha}
$$

are uniquely determined. Next we take

$$
\pi_{\lambda}=p_{\lambda \mu}\left(x^{i}\right) d x^{\mu}
$$

such that

$$
g_{\wedge \mu}\left(x^{i}\right) d x^{\lambda} d x^{\mu}=\sum\left(\pi_{\lambda}\right)^{2}
$$

Putting

$$
\pi_{\alpha}=a\left(x^{i}\right) \omega_{\alpha}
$$

we get from (1)

$$
d s^{2}=\sum\left(\pi_{i}\right)^{2}
$$

For these $\pi_{i}$ there exist uniquely determined Pfaffians $\pi_{i j}$ such that

$$
\pi_{i}^{\prime}=\left[\pi_{j}, \pi_{j i}\right], \quad \pi_{i j}=-\pi_{j i} .
$$

We get by (3) and (5)

$$
\pi_{\alpha}^{\prime}=\left(a \omega_{\alpha}\right)^{\prime}=\left[d a, \omega_{\alpha}\right]+a \omega_{\alpha}^{\prime}=\left[d a, \omega_{\alpha}\right]+a\left[\omega_{\beta}, \omega_{\beta \alpha}\right] .
$$

Then by (6)

$$
\left[d a, \omega_{\alpha}\right]+a\left[\omega_{\beta}, \omega_{\beta \alpha}\right]=\left[\pi_{\beta}, \pi_{\beta \alpha}\right]+\left[\pi_{\lambda}, \pi_{\lambda_{\alpha}}\right] .
$$

Taking (5) into consideration we have

$$
\left[\delta_{\alpha \beta} \frac{d a}{a}+\omega_{\alpha \beta}-\pi_{\alpha \beta}, \pi_{\beta}\right]+\left[-\pi_{\alpha \lambda}, \pi_{\lambda}\right]=0 .
$$

As $\pi_{\beta}$ 's and $\pi_{\lambda}$ 's are linearly independent, we can put

$$
\begin{gathered}
\delta_{\alpha \beta} \frac{d a}{a}+\omega_{\alpha \beta}-\pi_{\alpha \beta}=C_{\alpha \beta i} \pi_{i} \\
-\pi_{\alpha \lambda}=C_{\alpha \lambda i} \pi_{i}
\end{gathered}
$$

where

$$
C_{\alpha i j}=C_{\alpha j i} \text {. }
$$

As $\omega_{\alpha \beta}$ 's and $\pi_{\alpha \beta}$ 's are skew symmetric with respect to indices $\alpha$ and $\beta$ we get for $\alpha \neq \beta$

$$
C_{\alpha \beta i}+C_{\beta a i}=0 \quad(\alpha \neq \beta) .
$$

For $\alpha \neq \beta \neq \gamma$ we get by (9) and (10)

$$
C_{\alpha \beta \gamma}=-C_{\beta \alpha \gamma}=-C_{\beta \gamma \alpha}=C_{\gamma \beta \alpha}=C_{\gamma \alpha \beta}=-C_{\alpha \gamma \beta}=-C_{\alpha \beta \gamma} .
$$

Hence

$$
C_{\alpha \beta \gamma}=0 \quad(\alpha \neq \beta \neq \gamma) .
$$

For $\alpha=\beta$ we obtain by (7) 


$$
\frac{d a}{a}=C_{\alpha \propto i} \pi_{i}
$$

As $\pi_{i}$ 's are linearly independent $d a / a$ can be written uniquely as a linear combination of $\pi_{i}$ 's. So $C_{\alpha \alpha i}$ 's depend only on $i$ and not on $\alpha$. We put $C_{\alpha \alpha i}=C_{i}$ and we have

$$
\frac{d a}{a}=C_{i} \pi_{i}
$$

By (9) and (10)

$$
C_{\alpha \beta \beta}=-C_{\beta \alpha \beta}=-C_{\beta \beta \alpha}=-C_{\alpha}=-C_{\alpha \alpha \alpha} \quad(\alpha \neq \beta),
$$

and by (7)

$$
\pi_{\alpha \beta}=\omega_{\alpha \beta}-C_{\alpha \beta i} \pi_{i}
$$

Taking (11) and (13) into consideration we have

$$
\pi_{\alpha \beta}=\omega_{\alpha \beta}-C_{\beta} \pi_{\alpha}+C_{\alpha} \pi_{\beta}-C_{\alpha \beta \lambda} \pi_{\lambda} .
$$

Next by (6) and (8) we have

$$
\begin{aligned}
\pi_{\lambda}^{\prime} & =\left[\pi_{\alpha}, \pi_{\alpha \lambda}\right]+\left[\pi_{\mu}, \pi_{\mu \lambda}\right] \\
& =\left[\pi_{\alpha},-C_{\alpha \lambda \beta} \pi_{\beta}-C_{\alpha \lambda \nu} \pi_{\nu}\right]+\left[\pi_{\mu}, \pi_{\mu \lambda}\right] \\
& =-C_{\alpha \lambda_{\beta}}\left[\pi_{\alpha}, \pi_{\beta}\right]-C_{\alpha \lambda \nu}\left[\pi_{\alpha}, \pi_{\nu}\right]+\left[\pi_{\mu}, \pi_{\mu \lambda}\right] .
\end{aligned}
$$

Considering (2) and (4) and remarking that the terms $\left[d x^{\alpha}, d x^{\beta}\right]$ appear only in $C_{\alpha \lambda_{\beta}}\left[\pi_{\alpha}, \pi_{\beta}\right]$ we get $C_{\alpha \lambda \beta}=C_{\beta \lambda \alpha}$. Then by (9) $C_{\alpha \beta \lambda}=C_{\beta \alpha \lambda}$, while according to (10) $C_{\alpha \beta \lambda}+C_{\beta \alpha \lambda}=0$, and we get

$$
C_{\alpha \beta \lambda}=0 \quad(\alpha \neq \beta) .
$$

Then by (8)

$$
\begin{aligned}
\pi_{\alpha \lambda} & =-C_{\alpha \lambda i} \pi_{i}=-C_{\alpha \lambda \beta} \pi_{\beta}-C_{\alpha \lambda \mu} \pi_{\mu} \\
& =-C_{\alpha \lambda \alpha} \pi_{\alpha}-C_{\alpha \lambda \mu} \pi_{\mu} \\
& =-C_{\alpha \alpha \lambda} \pi_{\alpha}-C_{\alpha \lambda \mu} \pi_{\mu} .
\end{aligned}
$$

As $C_{\alpha \alpha \lambda}=C_{\lambda}$ we have

$$
\pi_{\alpha \lambda}=-C_{\lambda} \pi_{\alpha}-C_{\alpha \lambda \mu} \pi_{\mu} .
$$

Thus we obtain the following relations by (12), (14), (15) and $C_{\alpha \beta \lambda}=0$;

$$
\left\{\begin{array}{l}
\pi_{\alpha \beta}=\omega_{\alpha \beta}-C_{\beta} \pi_{\alpha}+C_{\alpha} \pi_{\beta} \\
\pi_{\alpha \lambda}=-C_{\lambda} \pi_{\alpha}-C_{\alpha \lambda \mu} \pi_{\mu} \\
\frac{d a}{a}=C_{i} \pi_{i} .
\end{array}\right.
$$

2. Now we introduce a Riemannian connection into our space by

$$
d \mathbf{A}=\pi_{i} \mathbf{e}_{i}, \quad d \mathbf{e}_{i}=\pi_{i j} \mathbf{e}_{j}
$$


and develop the tangent euclidean spaces on a euclidean space. Then for a point $\overline{\mathbf{A}}=\mathbf{A}+t_{\lambda} \mathbf{e}_{\lambda}$ we have

$$
\begin{aligned}
d \overline{\mathbf{A}} & =d \mathbf{A}+d t_{\lambda} \mathbf{e}_{\lambda}+t_{\lambda} d \mathbf{e}_{\lambda}=\pi_{i} \mathbf{e}_{i}+d t_{\lambda} \mathbf{e}_{\lambda}+t_{\lambda} \pi_{\lambda i} \mathbf{e}_{i} \\
& =\left(\pi_{\alpha}+t_{\lambda} \pi_{\lambda \alpha}\right) \mathbf{e}_{\alpha}+\left(\pi_{\lambda}+d t_{\lambda}+t_{\mu} \pi_{\mu \lambda}\right) \mathbf{e}_{\lambda} .
\end{aligned}
$$

When we develop along the $k$-dimensional surfaces $x^{\lambda}=$ const, we have by (4) $\pi_{\lambda}=0$. Then $d \mathbf{A}=\pi_{\alpha} \mathbf{e}_{\alpha}$ and

$$
\pi_{\alpha}+t_{\lambda} \pi_{\lambda \alpha}=\pi_{\alpha}+t_{\lambda}\left(C_{\lambda} \pi_{\alpha}+C_{\alpha \lambda_{\mu}} \pi_{\mu}\right)=\left(1+C_{\lambda} t_{\lambda}\right) \pi_{\alpha} .
$$

Now we assume $a\left(x^{i}\right)$ contains at least one $x^{\lambda}$. In this case, owing to (12), there exists among $C_{\lambda}$ 's at least one $C_{\lambda}$ which is not zero, and consequently there exist $t_{\lambda}$ 's such that

$$
1+C_{\lambda} t_{\lambda}=0 \text {. }
$$

When we develop along $k$-dimensional surfaces $x^{\lambda}=$ const, we get for the point A satisfying (17)

$$
d \overline{\mathbf{A}}=\left(d t_{\lambda}+t_{\mu} \pi_{\mu \lambda}\right) \mathbf{e}_{\lambda} .
$$

The points $\overline{\mathbf{A}}=\mathbf{A}+t_{\lambda} \mathbf{e}_{\lambda}$ for $t_{\lambda}$ 's satisfying (17) generate $n-k-1$-dimensional plane $P_{0}$ in the tangent euclidean space at $\mathbf{A}$ of the Riemann space (1). (18) indicates that when we develop along the $k$-dimensional surfaces $x^{\lambda}=$ const $\mathbf{A}$ is a fixed point or describes a curve touching to the $k$-dimensional plane $P$ spanned by the vectors $\mathbf{e}_{k+1}, \ldots, \mathbf{e}_{n}$ with $\mathbf{A}$ as their origin. Now we take $\mathbf{e}_{n}$ on the perpendicular from $\mathbf{A}$ to $P_{0}$. Then any point $\overline{\mathbf{A}}$ on $P_{0}$ can be represented by

$$
\overline{\mathbf{A}}=\mathbf{A}+t_{p} \mathbf{e}_{p}+t \mathbf{e}_{n}
$$

where $p$ runs from $k+1$ to $n-1$ and $t_{p}$ 's are arbitrary numbers, while $t$ is a fixed function of $x^{i}$ s. For the frame thus chosen the relations (A) hold and along surfaces $x^{\lambda}=$ const we have (18) and (17), and so

$$
1+C_{\lambda} t_{\lambda}=1+C_{p} t_{p}+C_{n} t=0
$$

holds for any $t_{p}$ 's $(p=k+1, \ldots, n-1)$. Thus

$$
C_{p}=0, \quad C_{n}=-\frac{1}{t} \text {. }
$$

Hence we get from (A)

$$
\left\{\begin{array}{l}
\pi_{\alpha p}=-C_{\alpha p \mu} \pi_{\mu}, \quad \pi_{\alpha n}=\frac{\pi_{\alpha}}{t}-C_{\alpha n \mu} \pi_{\mu} \\
\frac{d a}{a}=-\frac{\pi_{n}}{t}+C_{\alpha} \pi_{\alpha} .
\end{array}\right.
$$

If we put $x^{\alpha}=$ const, we get $\pi_{\alpha}=0$ and so $d \mathbf{A}=\pi_{\lambda} \mathbf{e}_{\lambda}$ and $\frac{d a}{a}=-\frac{\pi_{n}}{t}$. Thus we obtain 
THEOREM 1. Let the square of arc-element of a Riemann space be given by (1), where $a\left(x^{i}\right)$ actually contains at least one $x^{\lambda}$. Then there exist in the tan. gent euclidean space at any point $\mathbf{A}$ of the Riemann space an $n-k$-dimensional plane $P$ through $\mathbf{A}$ and an $n-k-1$-dimensional plane $P_{0}$ lying on Pand not passing through A, which have the following property;

$1^{\circ}$. If we develop along the k-dimensional surfaces $x^{\lambda}=$ const, $\mathbf{A}$ describes an arc perpendicular to $P$ at every instant and any point on $P_{0}$ is either a fixed point or describes an arc touching to $P$.

$2^{\circ}$. If we develop along $n-k$-dimensional surfaces $x^{\alpha}=$ const, $\mathbf{A}$ describes an arc touching to $P$, and when we denote the length of the normal from $\mathbf{A}$ to $P_{0}$ by $t$ and the orthogonal component of an arc-element of $\mathbf{A}$ in the direction of this normal by $\pi_{n}, \pi_{n} / t$ is a total differential of a certain function of $x^{\lambda}$ s.

3. Before stating the inverse of theorem 1 we prove a lemma.

Lemma. Let $\omega_{\alpha}(\alpha=1, \ldots, k)$ be linearly independent Pfaffians with $n$ variables. If there exist Pfaffians $\omega_{\alpha \beta}$ such that

$$
\omega_{\alpha}^{\prime}=\left[\omega_{\beta}, \omega_{\beta \alpha}\right], \quad \omega_{\alpha \beta}=-\omega_{\beta \alpha},
$$

then by a suitable choice of variables $x^{i}(i=1, \ldots, n)$ we have $\omega_{\alpha}=p_{\alpha \beta}\left(x^{i}\right) d x^{\beta}$, and for these variables $\sum\left(\omega_{\alpha}\right)^{2}$ does not contain $x^{\lambda}(\lambda=k+1, \ldots, n)$, namely $\sum\left(\omega_{\alpha}\right)^{2}=g_{\alpha \beta}\left(x^{\top}\right) d x^{\alpha} d x^{\beta}$.

The proof, which is quite natural, runs as follows. By Frobenius's theorem [2] p. 193 variables stated in the lemma exist. Writing the condition $\omega_{\alpha}^{\prime}=\left[\omega_{\beta}, \omega_{\beta \alpha}\right]$ more fully we have

$$
d \omega_{\alpha}(\delta)-\delta \omega_{\alpha}(d)=\omega_{\beta}(d) \omega_{\beta \alpha}(\delta)-\omega_{\beta}(\delta) \omega_{\beta \alpha}(d) .
$$

Let the symbols $d$ and $\delta$ be such that

$$
\left(d x^{1}, \ldots, d x^{k}, 0, \ldots, 0\right) \text { and }\left(0, \ldots, 0, \delta x^{k+1}, \ldots, \delta x^{n}\right) \text {. }
$$

Then

$$
-\delta \omega_{\alpha}(d)=\omega_{\beta}(d) \omega_{\beta \alpha}(\delta)
$$

And we have

$$
\delta\left(\sum\left(\omega_{\alpha}\right)^{2}\right)=2 \sum \omega_{\alpha} \delta \omega_{\alpha}=-2 \sum \omega_{\alpha} \omega_{\beta} \omega_{\beta \alpha}=0 .
$$

This shows $\sum\left(\omega_{\alpha}\right)^{2}$ does not contain $x^{\lambda}$.

Now the inverse of theorem 1 is

THEOREM 2. If an n-dimensional Riemann space has coordinate system $x^{i}$ in which the conditions $1^{\circ}$ and $2^{\circ}$ in theorem 1 hold, then the square of the arcelement of the space is of the form (1), a( $\left.x^{i}\right)$ containing at least one of $x^{\lambda}$ s. 
Proof. In the tangent euclidean space at $\mathbf{A}$ of the Riemann space we take a component $\mathbf{e}_{n}$ of an orthonormal system of vectors $\mathbf{e}_{1}, \ldots, \mathbf{e}_{n}$ on the perpendicular from $\mathbf{A}$ to $P_{0}$, and components $\mathbf{e}_{\lambda}(\lambda=k+1, \ldots, n)$ on $P$. Let the Riemannian connection be given by $d \mathbf{A}=\pi_{i} \mathbf{e}_{i}, d \mathbf{e}_{i}=\pi_{i j} \mathbf{e}_{j}$. By the condition $1^{\circ}$ we have $d \mathbf{A}=\pi_{\alpha} \mathbf{e}_{\alpha}$, which leads to $\pi_{\lambda}=0$. So in general

$$
\pi_{\lambda}=p_{\lambda \mu}\left(x^{i}\right) d x^{\mu} .
$$

For $\overline{\mathbf{A}}=\mathbf{A}+t_{\lambda} \mathbf{e}_{\lambda}$ we have by (16)

$$
d \overline{\mathbf{A}}=\left(\pi_{\alpha}+t_{\lambda} \pi_{\lambda \alpha}\right) \mathbf{e}_{\alpha}+\left(\pi_{\lambda}+d t_{\lambda}+t_{\mu} \pi_{\mu \lambda}\right) \mathbf{e}_{\lambda} .
$$

The latter half of $1^{\circ}$ shows that $d \overline{\mathbf{A}}$ does not contain $\mathbf{e}_{\alpha}$. for $x^{\lambda}=$ const and so

$$
\pi_{\alpha}+t_{\lambda} \pi_{\lambda \alpha}=0 \text {. }
$$

As $\overline{\mathrm{A}}$ moves on $P_{0}, t_{n}=t$ is constant, while $t_{k+1}, \ldots, t_{n-1}$ vary freely. So $\pi_{\alpha}+t \pi_{n \alpha}=0, \pi_{\alpha p}=0 \quad(p=k+1, \ldots, n-1)$. In general owing to (19) we obtain for suitable $C_{\alpha \lambda \mu}$

$$
\pi_{\alpha \lambda}=\delta_{n \lambda} \frac{\pi_{\alpha}}{t}-C_{\alpha \lambda \mu} \pi_{\mu}
$$

From the condition $2^{\circ} d \mathbf{A}=\pi_{\lambda} \mathbf{e}_{\lambda}$ along the surfaces $x^{\alpha}=$ const, and so $\pi_{\alpha}=0$. In general

$$
\pi_{\alpha}=p_{\alpha \beta}\left(x^{i}\right) d x^{\beta} .
$$

By the latter half of $2^{\circ}$ for $x^{\alpha}=$ const there exists a function $a=a\left(x^{i}\right)$ such that $\pi_{n} / t=-d a / a$. So in general

$$
\frac{\pi_{n}}{t}=-\frac{d a}{a}+C_{\beta} \pi_{\beta}
$$

where $a=a\left(x^{i}\right)$ and $C_{\beta}$ 's are suitable functions.

Let $\omega_{\alpha}=\pi_{\alpha} / a$. Then

$$
\begin{aligned}
\pi_{\alpha}^{\prime} & =\left[d a, \omega_{\alpha}\right]+a \omega_{\alpha}^{\prime}=\left[d a, \frac{\pi_{\alpha}}{a}\right]+a \omega_{\alpha}^{\prime} \\
& =\left[\frac{d a}{a}, \pi_{\alpha}\right]+a \omega_{\alpha}^{\prime}=\left[-\frac{\pi n}{t}+C_{\beta} \pi_{\beta}, \pi_{\alpha}\right]+a \omega_{\alpha}^{\prime} .
\end{aligned}
$$

By (20)

$$
\begin{aligned}
{\left[\pi_{i}, \pi_{i \alpha}\right] } & =\left[\pi_{\beta}, \pi_{\beta \alpha}\right]+\left[\pi_{\lambda}, \pi_{\lambda \alpha}\right] \\
& =\left[\pi_{\beta}, \pi_{\beta \alpha}\right]+\left[\pi_{\lambda}, C_{\alpha \lambda \mu} \pi_{\mu}-\delta_{n \lambda} \frac{\pi_{\alpha}}{t}\right] \\
& =\left[\pi_{\beta}, \pi_{\beta \alpha}\right]+C_{\alpha \lambda \mu}\left[\pi_{\lambda}, \pi_{\mu}\right]-\frac{1}{t}\left[\pi_{n}, \pi_{\alpha}\right] .
\end{aligned}
$$

As $\pi_{\alpha}^{\prime}=\left[\pi_{i} \pi_{i \alpha}\right]$, we have

$$
a \omega_{\alpha}^{\prime}=\left[\pi_{\beta}, \pi_{\beta \alpha}\right]-C_{\beta}\left[\pi_{\beta}, \pi_{\alpha}\right]+C_{\alpha \lambda \mu}\left[\pi_{\lambda}, \pi_{\mu}\right] .
$$


Here by (21) and $\pi_{\alpha}=a \omega_{\alpha}$ there are not terms $\left[d x^{\lambda}, d x^{\mu}\right]$ on the left side $a \omega_{\alpha}^{\prime}$ $=q_{i j}\left[d x^{i}, d x^{j}\right]$, while on the right side $C_{\alpha \lambda \mu}\left[\pi_{\lambda}, \pi_{\mu}\right]=\gamma_{\lambda \mu}\left[d x^{\lambda}, d x^{\mu}\right]$. So the last terms must be zero, namely $C_{\alpha \lambda \mu}=C_{\alpha \mu \lambda}$. We have by (23)

$$
\omega_{\alpha}^{\prime}=\left[\omega_{\beta}, \pi_{\beta \alpha}\right]-C_{\beta}\left[\omega_{\beta}, \pi_{\alpha}\right] \text {. }
$$

Then putting

$$
\omega_{\beta \alpha}=\pi_{\beta \alpha}-C_{\beta} \pi_{\alpha}+C_{\alpha} \pi_{\beta}
$$

we have

$$
\omega_{\alpha}^{\prime}=\left[\omega_{\beta}, \omega_{\beta \alpha}\right] \text {. }
$$

As $\omega_{\alpha \beta}$ are shew symmetric with respect to indices $\alpha$ and $\beta$, we have by lemma $\sum\left(\omega_{\alpha}\right)^{2}=g_{\alpha \beta}\left(x^{\gamma}\right) d x^{\alpha} d x^{\beta}$. Thus

$$
\begin{aligned}
d s^{2} & =\sum\left(\pi_{i}\right)^{2}=\sum\left(\pi_{\alpha}\right)^{2}+\sum\left(\pi_{\curlywedge}\right)^{2}=\sum\left(a \omega_{\alpha}\right)^{2}+\sum\left(\pi_{\lambda}\right)^{2} \\
& =a\left(x^{i}\right)^{2} g_{\alpha \beta}\left(x^{\curlyvee}\right) d x^{\alpha} d x^{\beta}+g_{\lambda \mu}\left(x^{i}\right) d x^{\lambda} d x^{\mu} .
\end{aligned}
$$

By (22) $a\left(x^{i}\right)$ contains at least one of $x^{\lambda}$ s.

4. Now we return to 2 and treat the case in which $a\left(x^{i}\right)$ in (1) does not contain any $x^{\lambda}$. This case reduces to the case $a\left(x^{i}\right)=1$. When $a\left(x^{i}\right)=1$, we have by (12) $C_{i}=0$ and by (15) $\pi_{\alpha \lambda}=-C_{\alpha \lambda \mu} \pi_{\mu}$. Along surfaces $x^{\lambda}=$ const we have $\pi_{\alpha \lambda}=0$ and consequently $d \mathbf{e}_{\alpha}=\pi_{\alpha \beta} \mathbf{e}_{\beta}$. Hence

THEOREM 3. If the square of arc element of an n-dimensional Riemann space is for a suitably chosen coordinate system $x^{i}$

$$
d s^{2}=g_{\alpha \beta}\left(x^{\gamma}\right) d x^{\alpha} d x^{\beta}+g_{\lambda \mu}\left(x^{i}\right) d x^{\lambda} d x^{\mu},
$$

then there exists in a tangent euclidean space at any point $\mathbf{A}$ of the Riemann space an $n$-k-dimensional plane $P$ satisfying the following conditions:

$1^{\circ}$. If we develop along $k$-dimensional surfaces $x^{\lambda}=$ const, $\mathbf{A}$ describes an arc which is perpendicular to $P$ at any instant, and $P$ moves parallel to a fixed $n$-k-dimensional plane.

$2^{\circ}$. If we develop along $n$-k-dimensional surfaces $x^{\alpha}=$ const, $\mathbf{A}$ describes an arc which is parallel to $P$.

The inverse of this theorem is also true.

THEOREM 4. If two conditions $1^{\circ}$ and $2^{\circ}$ in theorem 3 hold for suitably chosen coordinates $x^{i}$ of an n-dimensional Riemann space, then the square of arc element of our space is of the form

$$
d s^{2}=g_{\alpha \beta}\left(x^{\top}\right) d x^{\alpha} d x^{\beta}+g_{\lambda \mu}\left(x^{i}\right) d x^{\lambda} d x^{\mu} .
$$

Proof. In the tangent euclidean space at $\mathbf{A}$ of our Riemann space we take $\mathbf{e}_{\lambda}(\lambda=k+1, \ldots, n)$ on the plane $P$. Then by the condition $1^{\circ}$ we have $\pi_{\lambda}=0$ 
along surfaces $x^{\lambda}=$ const, and in general

$$
\pi_{\lambda}=p_{\lambda \mu}\left(x^{i}\right) d x^{\mu} \text {. }
$$

The latter half of $1^{\circ}$ indicates $d \mathbf{e}_{\lambda}=\pi_{\lambda \mu} \mathbf{e}_{\mu}$ along $x^{\lambda}=$ const and consequently $\pi_{\alpha \lambda}=0$. So in general

$$
\pi_{\alpha \lambda}=-C_{\alpha \lambda \mu} \pi_{\mu}
$$

By the condition $2^{\circ}$

$$
\pi_{\alpha}=p_{\alpha \beta}\left(x^{i}\right) d x^{\beta}
$$

By (26) and (6)

$$
\pi_{\alpha}^{\prime}=\left[\pi_{\beta}, \pi_{\beta \alpha}\right]+\left[\pi_{\lambda}, \pi_{\lambda \alpha}\right]=\left[\pi_{\beta}, \pi_{\beta \alpha}\right]+C_{\alpha \lambda \mu}\left[\pi_{\lambda}, \pi_{\mu}\right] .
$$

Taking (25) and (27) into consideration we get $C_{\alpha \lambda_{\mu}}\left[\pi_{\lambda}, \pi_{\mu}\right]=0$ and we have $\pi_{\alpha}^{\prime}=\left[\pi_{\beta}, \pi_{\beta \alpha}\right]$ and by lemma $\sum\left(\pi_{\alpha}\right)^{2}=g_{\alpha \beta}\left(x^{\gamma}\right) d x^{\alpha} d x^{\beta}$.

Remark. In place of $1^{\circ}$ and $2^{\circ}$ in theorem 4 we take the following two conditions :

$1^{\circ}$. If we develop along $k$-dimensional surfaces $x^{\lambda}=$ const, $P$ moves parallel to a fixed $n$ - $k$-dimensional plane.

$2^{\circ}$. If we develop along $n-k$-dimensional surfaces $x^{\alpha}=$ const, $P$ moves parallel to a fixed $n$ - $k$-dimensional plane.

Then by $1^{\circ} \pi_{\alpha \lambda}=0$ for $x^{\lambda}=$ const and by $2^{\circ} \pi_{\alpha \lambda}=0$ for $x^{\alpha}=$ const. So in general $\pi_{\alpha \lambda}=0$, and by (6)

$$
\begin{aligned}
& \pi_{\alpha}^{\prime}=\left[\pi_{i}, \pi_{i \alpha}\right]=\left[\pi_{\beta}, \pi_{\beta \alpha}\right]+\left[\pi_{\lambda}, \pi_{\lambda \alpha}\right]=\left[\pi_{\beta}, \pi_{\beta \alpha}\right] \\
& \pi_{\lambda}^{\prime}=\left[\pi_{i}, \pi_{i \lambda}\right]=\left[\pi_{\alpha}, \pi_{\alpha \lambda}\right]+\left[\pi_{\mu}, \pi_{\mu \lambda}\right]=\left[\pi_{\mu}, \pi_{\mu \lambda}\right] .
\end{aligned}
$$

Then by lemma $\sum\left(\pi_{\alpha}\right)^{2}=g_{\alpha_{\beta}}\left(y^{\curlyvee}\right) d y^{\alpha} d y^{\beta}$ for suitably chosen coordinates $y^{i}=y^{i}\left(x^{j}\right)$ and $\sum\left(\pi_{\lambda}\right)^{2}=g_{\lambda \mu}\left(z^{\nu}\right) d z^{\lambda} d z^{\mu}$ for suitably chosen coordinates $z^{i}=z^{i}\left(x^{j}\right)$. If we take $u^{\alpha}=y^{\alpha}\left(x^{j}\right), u^{\lambda}=z^{\lambda}\left(x^{j}\right)$ as coordinates we get

$$
d s^{2}=\sum\left(\pi_{i}\right)^{2}=g_{\alpha \beta}\left(u^{\gamma}\right) d u^{\alpha} d u^{\beta}+g_{\lambda \mu}\left(u^{\nu}\right) d u^{\lambda} d u^{\mu} .
$$

This space has been treated by many authors.

5. Now we treat the special case $k=n-1$. In this case the condition $2^{\circ}$ in theorem 2 is unnecessary. The second part of $2^{\circ}$, namely the condition that $\pi_{n} / t$ is a total differential for $x^{\alpha}=$ const, is always satisfied. We will prove the first part of $2^{\circ}$ is also satisfied under the condition $1^{\circ}$. By 1 the equations (20) hold, which in this case reduce to $\pi_{\alpha n}=\pi_{\alpha} / t-C_{\alpha n n} \pi_{n}$. Consequently

$$
\begin{aligned}
\pi_{\alpha}^{\prime} & =\left[\pi_{\beta}, \pi_{\beta \alpha}\right]+\left[\pi_{n}, \pi_{n \alpha}\right]=\left[\pi_{\beta}, \pi_{\beta \alpha}\right]+\left[\pi_{n},-\frac{\pi_{\alpha}}{t}+C_{\alpha n n} \pi_{n}\right] \\
& =\left[\pi_{\beta}, \pi_{\beta \alpha}+\delta_{\beta \alpha} \frac{\pi_{n}}{t}\right]
\end{aligned}
$$




$$
\pi_{n}^{\prime}=\left[\pi_{\alpha}, \pi_{\alpha n}\right]=\left[\pi_{\alpha}, \frac{\pi_{\alpha}}{t}-C_{\alpha n n} \pi_{n}\right]=C_{\alpha n n}\left[\pi_{n}, \pi_{\alpha}\right]
$$

By Frobenius's theorem [2] p. 193 for suitably chosen coordinates $y^{\alpha}=y^{\alpha}\left(x^{j}\right)$ and $y^{n}=y^{n}\left(x^{j}\right)$ we have

$$
\pi_{\alpha}=p_{\alpha \beta}\left(y^{i}\right) d y^{\beta}, \quad \pi_{n}=p\left(y^{i}\right) d y^{n} .
$$

As $\pi_{n}=0$ for $x^{n}=$ const by (19), $y^{n}$ is a function of only one variable $x^{n}$. For coordinate system $y^{i}$ thus chosen the first part of $2^{\circ}$ is satisfied, and we have

THEOREM 5. The necessary and sufficient condition for an n-dimensional Riemann space to have an arc element which can be writen in the form

$$
\begin{aligned}
& d s^{2}=a\left(x^{i}\right)^{2} g_{\alpha_{\beta}}\left(x^{\top}\right) d x^{\alpha} d x^{\beta}+g_{n n}\left(x^{i}\right)\left(d x^{n}\right)^{2} \\
&(\alpha, \beta, \gamma=1,2, \ldots, n-1)
\end{aligned}
$$

is that there exists a point $P_{0}$ in the tangent euclidean space at any point $\mathbf{A}$ of the Riemann space which has the property that, if we develop along any hypersurface of one-parametric family of hypersurfaces, $\mathbf{A}$ describes an arc perpendicular to $\mathbf{A} P_{0}$ and $P_{0}$ describes an arc which touches the straight line $\mathbf{A} P_{0}$ or is a fixed point.

Especially we treat the case $g_{n n}\left(x^{i}\right)=g_{n n}\left(x^{n}\right)$, which reduces to $g_{n n}\left(x^{i}\right)=1$. Then in 2 we have $\pi_{n}=d x^{n}$ and consequently $\pi_{n}^{\prime}=\left[\pi_{\alpha}, \pi_{a n}\right]=0$.

By (B) we have $\pi_{\alpha n}=\pi_{\alpha} / t-C_{\alpha n n} \pi_{n}$. So $-C_{\alpha n n}\left[\pi_{\alpha}, \pi_{n}\right]=0$ and hence $C_{\alpha n n}=0$. Thus

$$
\pi_{a n}=\frac{\pi_{n}}{t}
$$

By (16) we have

$$
d\left(\mathbf{A}+t \mathbf{e}_{n}\right)=\left(\pi_{\alpha}-t \pi_{\alpha n}\right) \mathbf{e}_{\alpha}+\left(\pi_{n}+d t\right) \mathbf{e}_{n} .
$$

So on account of (28)

$$
d\left(\mathbf{A}+t \mathbf{e}_{n}\right)=\left(\pi_{n}+d t\right) \mathbf{e}_{n} .
$$

Thus by development the point $\boldsymbol{P}_{0}=\mathbf{A}+t \mathbf{e}_{n}$ is a fixed point or describes an arc touching to $\mathbf{A} P_{0}$.

Conversely we assume there is a point $P_{0}$ in the tangent euclidean space of any point $\mathbf{A}$ of Riemann space which has the property that by development $P_{0}$ is a fixed point or describes an arc touching to $A P_{0}$. Then (30) holds and (28) holds by virtue of (29). Consequently $\pi_{n}^{\prime}=\left[\pi_{n \alpha}, \pi_{\alpha}\right]=0$ and $\pi_{n}$ is a total differential of a certain function. Putting $\pi_{n}=d x^{n}$ we proceed as in the proof of theorem 2. Hence 
THEOREM 6. The necessary and sufficient condition for a Riemann space to have an arc-element such that

$$
d s^{2}=a\left(x^{i}\right)^{2} g_{\alpha \beta}\left(x^{\gamma}\right) d x^{\alpha} d x^{\beta}+\left(d x^{n}\right)^{2} \quad(\alpha, \beta, \gamma=1,2, \ldots, n-1)
$$

is that there is a point $P_{0}$ in the tangent euclidean space at any point $\mathbf{A}$ of the Riemann space such that by development $P_{0}$ is a fixed point or describes an arc touching to $\mathbf{A} P_{0}$.

This case has been proved by $\mathrm{K}$. Yano (the so-colled Riemann space with torse-forming vector field). If $P_{0}$ is a fixed point we have by (30) $\pi_{n}+d t=0$ and then by 2 (B) $d a / a=d t / t+C_{\alpha} \pi_{\alpha}$, namely $a=c t, c$ being a function of $x^{\alpha}$. So this case reduces to the case $a\left(x^{i}\right)=x^{n}$. The inverse is also true.

If $k=n-1$ in theorem 4 , the condition $2^{\circ}$ is also unnecessary. Hence

THEOREM 7. The necessary and sufficient condition for the Riemann space to have an arc-element given by

$$
d s^{2}=g_{\alpha \beta}\left(x^{\gamma}\right) d x^{\alpha} d x^{\beta}+g_{n n}\left(x^{i}\right)\left(d x^{n}\right)^{2} \quad(\alpha, \beta, \gamma=1,2, \ldots, n-1)
$$

is that there exists $a$ direction $P$ in the tangent euclidean space at any point of the Riemann space such that if we develop along any hypersurface of one-parametric family of hypersurfaces $P$ moves parallel to a fixed direction.

The special case in which $g_{n n}\left(x^{i}\right)=g_{n n}\left(x^{n}\right)$ holds reduces to the case $g_{n n}\left(x^{i}\right)$ $=1$, which is treated in the remark of theorem 4 .

\section{REFERENCES}

[1] K. Yano: Conformally separable quadratic differential form, Proc. Imp. Acad. Tokyo, Vol. 16, 1940.

[2] E. Cartan: La théorie des groupes finis et continus et la géometrie différentielles, 1937.

Mathematical Institute, Nagoya University 